\title{
Default mode network subsystem alterations in obsessive-compulsive disorder
}

\author{
Jan C. Beucke, Jorge Sepulcre, Mark C. Eldaief, Miriam Sebold, Norbert Kathmann \\ and Christian Kaufmann
}

\section{Background}

Although neurobiological models of obsessive-compulsive disorder (OCD) traditionally emphasise the central role of corticostriatal brain regions, studies of default mode network integrity have garnered increasing interest, but have produced conflicting results.

\section{Aims \\ To resolve these discrepant findings by examining the integrity of default mode network subsystems in OCD.}

\section{Method}

Comparison of seed-based resting-state functional connectivity of 11 default mode network components between 46 patients with $O C D$ and 46 controls using functional magnetic resonance imaging.

\section{Results}

Significantly reduced connectivity within the dorsal medial prefrontal cortex self subsystem was identified in the OCD group, and remained significant after controlling for medication status and life-time history of affective disorders. Further, greater connectivity between the self subsystem and salience and attention networks was observed.

\section{Conclusions}

Results indicate that people with OCD show abnormalities in a neural system previously associated with self-referential processing in healthy individuals, and suggest the need for examination of dynamic interactions between this default mode network subsystem and other large-scale networks in this disorder.

\section{Declaration of interest} None.
Neurobiological models of obsessive-compulsive disorder (OCD), a disabling neuropsychiatric disease characterised by intrusive, ego-dystonic obsessive thoughts and compulsive and/or ritualistic behaviours, ${ }^{1}$ traditionally emphasise corticostriatal brain abnormalities. ${ }^{2}$ In support of this assumption, recent resting-state functional connectivity magnetic resonance imaging (rs-fcMRI) studies have identified corticostriatal connectivity abnormalities in patients with OCD. ${ }^{3,4}$ However, consistent evidence of additional alterations in non-corticostriatal regions ${ }^{5}$ has led to doubts about whether the existing models can fully explain the clinical phenotype of OCD. ${ }^{5}$ At the same time, clinical applications of rs-fcMRI, testing the integrity of large-scale brain systems such as the default mode, ${ }^{6}$ executive control, ${ }^{7}$ dorsal attention $^{8}$ and salience ${ }^{7}$ networks in neurological and psychiatric disorders, have received increasing interest, and recent network models of psychiatric disorders hypothesise abnormalities in default mode, salience and executive control network functioning. ${ }^{9,10}$ Accordingly, potential abnormalities in the default mode, salience and the executive control networks represent an emerging topic of interest in studies of patients with OCD. ${ }^{11-15}$

Whereas elevated neural responses to errors ${ }^{11,16}$ or conflict $^{17}$ in components of the salience network such as the cingulate cortex and/or the anterior insula are consistent with the view that the salience network is hyperactive in OCD, ${ }^{9}$ the extant investigations of default mode network integrity have been conflicting. More precisely, these analyses have either identified connectivity abnormalities within the default mode network, or aberrant connectivity between the default mode network and components of other functional networks. Studies reporting abnormalities within the default mode network include seed-based rs-fcMRI examinations of core components in this network, such as the posterior cingulate cortex and/or anterior medial prefrontal cortex (aMPFC), as well as independent component analysis of experimental fMRI data ${ }^{12}$ revealing significant connectivity reductions ${ }^{11,12}$ and trends for lower connectivity ${ }^{14}$ in this network in patients with OCD. Studies reporting no connectivity abnormalities within the default mode network, but aberrant connectivity between this network and other functional networks include seed-based rs-fcMRI examinations of the posterior cingulate cortex ${ }^{13}$ and the anterior insula ${ }^{14}$ revealing reduced connectivity between posterior cingulate cortex and corticostriatal areas, ${ }^{13}$ and greater connectivity between the anterior insula and several default mode network components ${ }^{14}$ in patients with OCD. In addition to inter-network abnormalities involving the default mode network, greater connectivity between the anterior prefrontal cortex and insular and midcingulate areas, indicative of stronger connections between the executive control and the salience networks, has been found in rs-fcMRI studies of people with OCD. ${ }^{15}$

The present study sought to potentially resolve the disparities among existing default mode network findings in OCD through consideration of recent evidence that this network comprises a midline core constituted by hub regions in the anterior medial prefrontal and posterior cingulate cortices ${ }^{18}$ and further two functionally dissociable subsystems, one engaging in self-referential processes and the other involved in episodic memory. ${ }^{18}$ In contrast to previous rs-fcMRI studies in OCD, which exclusively tested for connectivity alterations of the midline core,${ }^{11,13,14}$ the present study sought to study the default mode network as a whole, thus additionally testing the integrity of the dorsomedial prefrontal cortex (dMPFC) self subsystem and medial temporal lobe memory subsystem in comparably large samples of patients with OCD (the OCD group) and demographically matched healthy controls (the control group). Based on previous findings of reduced default mode network connectivity in OCD, it was hypothesised that the OCD group would exhibit reduced connectivity in subsystems of the default mode network as compared with the control group. No specific subsystem hypothesis (i.e. reduced connectivity in one subsystem but not another) was formulated. Additionally, altered interconnectivity 
between the default mode network and other functional networks was explored, but given the inconsistency of previous findings, no hypotheses were formulated with respect to inter-network connectivity abnormalities.

\section{Method}

\section{Participants}

A total of 46 adult out-patients with a DSM-IV ${ }^{19}$ diagnosis of OCD were recruited from the OCD out-patient clinic at Humboldt-Universität zu Berlin and we also recruited 46 healthy controls. Pair-wise matching was performed with respect to demographic variables (age, gender, handedness and education). All participants were included in a previous analysis of this data-set. ${ }^{4}$ The OCD group were interviewed by a licensed clinical psychologist and diagnosed using the German version of the Structured Clinical Interview for DSM-IV (SCID). ${ }^{20}$ Comorbid lifetime diagnoses included major depression $(n=24)$, specific phobia $(n=3)$, hypochondriasis $(n=3)$, substance misuse $(n=2)$, social phobia $(n=7)$, dysthymia $(n=2)$, bulimia $(n=2)$, anorexia $(n=2)$, generalised anxiety disorder $(n=5)$, panic disorder $(n=4)$, somatoform disorder $(n=1)$, agoraphobia $(n=1)$ and adjustment disorder $(n=1)$. Nine patients had OCD as their single diagnosis. In the OCD group, 23 participants were treated with antidepressants $(20$ receiving selective serotonin reuptake inhibitors (SSRI), 2 receiving selective noradrenalin reuptake inhibitors (SNRI) and 1 treated with a tricyclic antidepressant). Unmedicated participants in the OCD group received no medication for OCD symptoms for at least 6 weeks prior to study participation. Table 1 displays demographic data for both the OCD and control groups. Exclusion criteria for participants included cardiac pacemakers or other metallic implants or artefacts and pregnancy. In the OCD group, there was no significant neurological illness, no prior neurosurgery, dementia, delirium, schizophrenia, delusional disorder or other psychotic disorder. None of the OCD group received benzodiazepines within 4 weeks prior to the imaging study. In the control group, participants did not fulfil criteria of any DSM-IV diagnosis as indicated by negative SCID screening questionnaires. All participants received a complete description of the study and written informed consent was obtained. The study was approved by the local ethics committee.

\section{Clinical scales and questionnaires}

Severity of OCD symptoms was evaluated using the German versions of the Yale-Brown Obsessive Compulsive Scale (Y-BOCS) ${ }^{21}$ and the Obsessive-Compulsive Inventory Revised (OCI-R). ${ }^{22}$ In addition, the State and Trait Anxiety Inventory-Revised (STAI-R), ${ }^{23}$ the Beck Depression Inventory (BDI), ${ }^{24}$ and the Montgomery-Åsberg Depression Rating Scale (MADRS) ${ }^{25}$ were administered prior to the fMRI study. The Edinburgh Handedness Inventory ${ }^{26}$ was used to classify handedness. In each group, there were 42 right-handed and three left-handed participants, and 1 person was ambidextrous. All participants completed a German vocabulary test ${ }^{27}$ as a measure of verbal intelligence.

\section{MRI procedures and preprocessing}

A total of 160 resting-state volumes $\left(T_{2}{ }^{*}\right.$-weighted single-shot gradient echo planar imaging sequence) was acquired on a $1.5 \mathrm{~T}$ Siemens Sonata system equipped with a circular-polarised headcoil using the following parameters: repetition time $(\mathrm{TR})=2000 \mathrm{~ms}$; echo time $(\mathrm{TE})=40 \mathrm{~ms} ; 35$ consecutive slices; isotropic $3 \mathrm{~mm}$ voxel size; flip angle, 90; field of view $(F O V)=192 \mathrm{~mm} ; 64 \times 64$ matrix, aligned parallel to the anterior-posterior commissure line and 176 anatomical slices were acquired using the Modified Driven Equilibrium Fourier Transform (MDEFT) sequence (spatial resolution $1 \times 1 \times 1 \mathrm{~mm}$; TR= $12.24 \mathrm{~ms}$;E $=3.56 \mathrm{~ms}$; flip angle, 23; $256 \times 224$ matrix). Participants were instructed to close their eyes, to relax and not to think of anything specific during this $5 \mathrm{~min}, 20 \mathrm{~s}$ run. Simultaneous electroencephalogram (32 channels with BrainAmp MR, Brain Products) was monitored online, and revealed that none of the participants fell asleep, which was also confirmed by a post-scan questionnaire. In order to reduce head motion, participants' heads were immobilised by vacuum head cushions. Earplugs were provided to attenuate background noise. Image preprocessing consisted of removal of the first four volumes, slice-time correction, motion correction and linear spatial normalisation to the atlas space of the Montreal Neurological Institute (MNI). Temporal filtering retained frequencies below $0.08 \mathrm{~Hz}$. Spurious or regionally non-specific variance was removed by regression of nuisance variables, including six-parameter rigid body head motion, the signal averaged over the whole brain (to account for respiratory effects), the lateral ventricles and over a region centred in the deep cerebral white matter. The fMRI data was then spatially smoothed using a $4 \mathrm{~mm}$ full-width at half maximum kernel.

\section{Default mode network subsystem functional connectivity (seed-to-voxel) analyses}

Standard rs-fcMRI functional connectivity analysis was performed for the left-lateralised 11 seed regions that were recently used to

\begin{tabular}{|c|c|c|}
\hline & $\begin{array}{l}\text { Obsessive-compulsive } \\
\text { disorder group }(n=46)\end{array}$ & $\begin{array}{l}\text { Control group } \\
\qquad(n=46)\end{array}$ \\
\hline Gender, female/male: $n$ & $26 / 20$ & $26 / 20$ \\
\hline Age, years: mean (s.d.) & $30.7(9.4)$ & $30.3(8.8)$ \\
\hline Education, years: mean (s.d.) & $12.0(1.6)$ & $12.1(1.5)$ \\
\hline IQ (verbal), mean (s.d.) & $106.2(9.6)$ & $107.1(9.8)$ \\
\hline \multicolumn{3}{|l|}{ State Trait Anxiety Inventory - revised version (STAI-R), mean (s.d.) } \\
\hline STAI-X1 (state) & $40.8(8.2)$ & $29.8(5.1)$ \\
\hline STAI-X2 (trait) & $49.3(10.3)$ & $30.6(7.2)$ \\
\hline Obsessive-Compulsive Inventory - revised version, mean (s.d.) & $23.9(11.0)$ & $4.3(3.8)$ \\
\hline Beck Depression Inventory, mean (s.d.) & $14.1(9.6)$ & $2.7(3.0)$ \\
\hline Yale-Brown Obsessive Compulsive Scale, mean (s.d.) & $20.8(6.5)$ & - \\
\hline Montgomery-Åsberg Depression Rating Scale, mean (s.d.) & $8.5(6.8)$ & - \\
\hline Interscan movement, mean (s.d.) & $0.07(0.03)$ & $0.07(0.03)$ \\
\hline
\end{tabular}


identify subsystems of the default mode network ${ }^{18}$ (online Fig. DS1(a), online Table DS1) using methods that were described in detail previously. ${ }^{18}$ Briefly, correlation maps for each of the 11 default mode network seeds (spheres of $8 \mathrm{~mm}$ in radius) were computed through correlation of regional time courses (averaged over all voxels within the seed region sphere) with every voxel in the brain. Subsequently, correlation maps were transformed to Fisher $z$-maps. Individual $z$-maps were then smoothed with a $8 \mathrm{~mm}$ kernel using Statistical parametric mapping (SPM8; Wellcome Department of Cognitive Neurology, London, UK (http://www.fil.ion.ucl.ac.uk/spm/)) and entered into random effects analyses comparing connectivity maps of each seed between the OCD and control groups (two-sample $t$-tests). The resulting $t$-maps indexing differences between the OCD and control groups were corrected for multiple comparisons at the cluster level using the statistical threshold of $P<0.0045$ (representing a Bonferronicorrected $P$-value adjusted for 11 comparisons after cluster-level correction) using Monte Carlo simulations for fMRI data as implemented in the AlphaSim program for Unix (http://afni.nimh. nih.gov/pub/dist/doc/program_help/AlphaSim.html). In order to exclude head motion as a potential confound affecting functional connectivity group differences, ${ }^{28}$ we calculated each participants mean interscan movement, and compared this measure between groups (Table 1). Detailed information regarding analyses exclusively characterising the averaged connectivity of all nodes constituting each subsystem (i.e. midline core: one connection; dMPFC self system: six connections, medial temporal lobe memory system: ten connections, online Fig. DS2(d)), additional confirmatory rs-fcMRI analyses comparing connectivity of previously identified seed regions for dorsal attention, executive control and salience network connectivity as well as correlational analyses is available in the online supplement DS1.

\section{Results}

\section{Default mode network subsystem functional connectivity analyses}

Seed-to-voxel fcMRI analyses allowed robust identification of connectivity within the subsystems of the default mode network
$\left(P_{\mathrm{FWE}}<0.05\right.$, corrected for multiple comparisons, online Fig. 1(b)-(d), online Table DS3). In the midline core, the posterior cingulate cortex seed revealed significantly $(P<0.0045$, clustercorrected) reduced connectivity in the OCD group with the aMPFC and the right posterior cingulate cortex (control group $>$ OCD group, online Fig. DS2(a)), whereas higher (OCD group $>$ control group) connectivity was evident with the fusiform gyrus (online Fig. DS3(a)). In the dMPFC self subsystem, significantly reduced connectivity between lateral temporal cortex seed and $\mathrm{dMPFC}$ was evident in the OCD group (control group $>$ OCD group, online Fig. DS2(b)). Further, the temporal pole seed revealed significantly reduced connectivity with anterior cingulate cortex and aMPFC in the OCD group (control group $>$ OCD group, online Fig. DS2(c)) whereas higher connectivity was evident with the superior parietal lobule and the precuneus (OCD group $>$ control group, online Fig. DS3(c)). In addition, the dMPFC seed showed higher connectivity with the anterior insula in the OCD group (OCD group > control group, online Fig. DS3(b)). Additional slices displaying group differences with respect to connectivity of dMPFC self subsystem seeds are available in online Fig. DS4(b). Regarding the medial temporal lobe memory subsystem seeds, no significant group differences were found in components of default mode network subsystems. Reduced connectivity was evident between the parahippocampal cortex seed and superior parietal regions and the precuneus (control group > OCD group, online Fig. DS4(c)), whereas higher connectivity was found between the posterior inferior parietal lobule seed and the lingual gyrus, the retrosplenial cortex seed and the inferior frontal gyrus, and the hippocampal formation seed and superior temporal gyrus (OCD group > control group, online Fig. DS3(d)-(f)). All seed-to-voxel rs-fcMRI group differences are summarised in Table 2.

\section{Effect of medication}

Potential effects of medication on seed-to-voxel results were addressed by separate comparisons of unmedicated and medicated participants in the OCD group with the control group, and by direct comparisons of unmedicated and medicated participants with OCD (online Table DS4). This set of analyses revealed

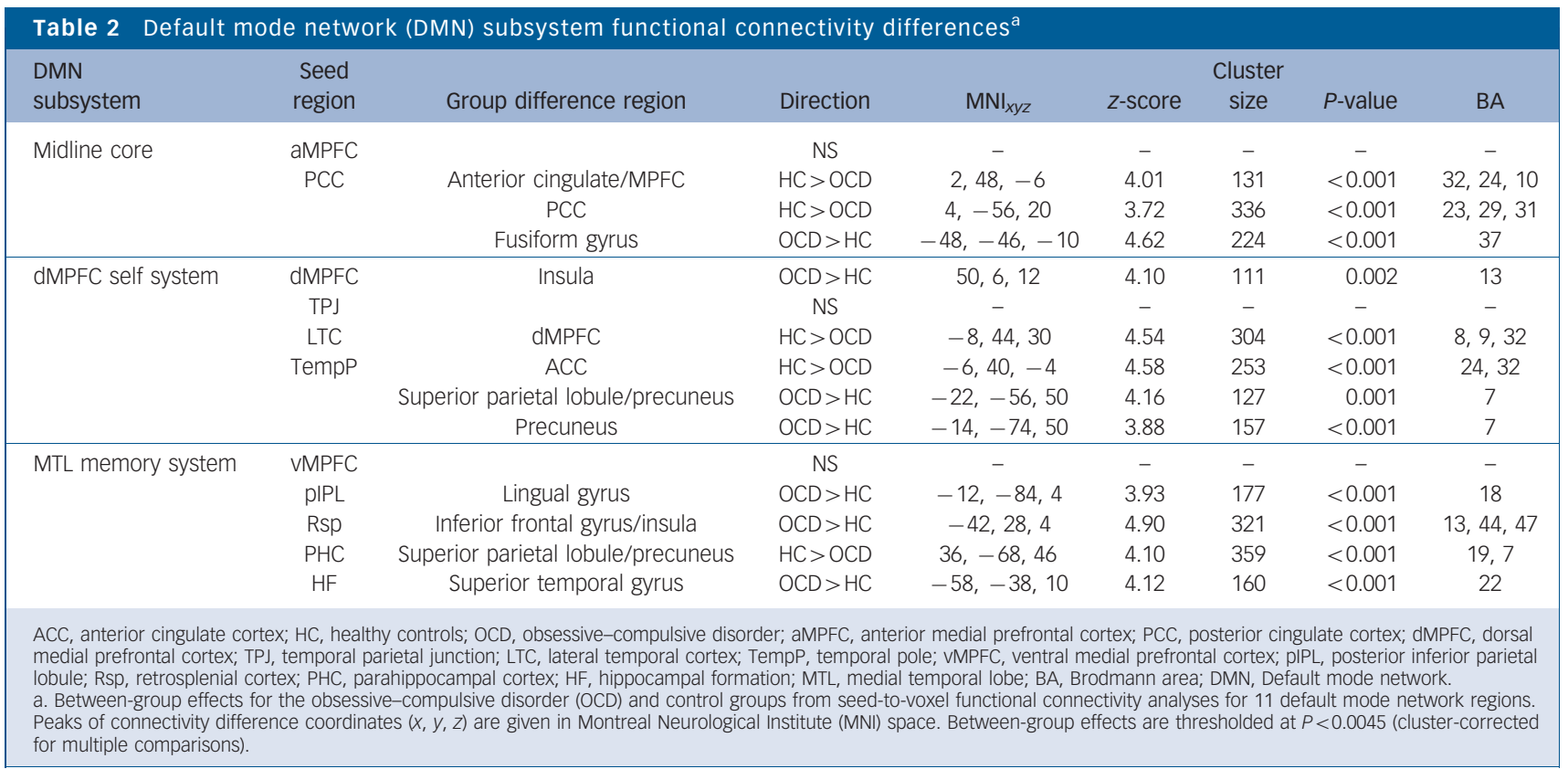


significantly reduced connectivity in the dMPFC self subsystem in unmedicated participants with OCD $(n=23)$ as compared with the control group $(n=23)$ (online Table DS4(a)), no significant differences between medicated participants with OCD and the control group in default mode network regions (online Table DS4(b)) and no differences between unmedicated and medicated participants with OCD in components of the default mode network (online Table DS4(c)).

\section{Effect of history of affective disorders}

Seed-to-voxel analyses were repeated after exclusion of participants in the OCD group with a lifetime history of affective disorders and the respective control group, revealing significantly reduced connectivity in the dMPFC self subsystem in the remaining OCD group participants $(n=18)$ in comparison with those in the control group $(n=18)$ (online Table DS5).

\section{Confirmatory functional connectivity (seed-to-voxel) analyses for dorsal attention, salience and executive control networks}

Additional seed-to-voxel fcMRI analyses comparing connectivity of dorsal attention, executive control and salience network seeds were conducted. The dorsal attention network seed in the left superior parietal lobule revealed higher connectivity with areas of the midline core and $\mathrm{dMPFC}$ self system in the OCD group compared with the control group, such as the anterior medial prefrontal cortex, the posterior cingulate cortex and the temporal pole (OCD group > control group, online Fig. DS5(a)), whereas the precentral gyrus showed reduced connectivity with the superior parietal lobule seed (online Table DS2). The salience network seed in the anterior insula exhibited greater connectivity with lateral temporal cortex and parahippocampal cortex in the OCD group compared with the control group (online Fig. DS5(b)), and lower connectivity with the inferior parietal lobule in the reverse contrast (control group $>$ OCD group, online Table DS2). The executive control network seed in the dorsolateral prefrontal cortex revealed greater connectivity with superior temporal areas approaching lateral temporal cortex in the OCD group compared with the control group (online Fig. DS5(c)), whereas dorsolateral prefrontal cortex connectivity with the inferior frontal gyrus was reduced in the OCD group compared with the control group (online Table DS2).

\section{Correlational analyses between dMPFC connectivity and OCD symptom severity}

Significant positive relationships were found between global OCD symptom severity as measured by the Y-BOCS (total score) and $\mathrm{dMPFC}$ connectivity with the anterior cingulate cortex $\left(\mathrm{MNI}_{x y z}=-8,36,30, z=4.61, r=0.62\right)$ and the ventral striatum $\left(\mathrm{MNI}_{x y z}=16,12,-2, z=3.98, r=0.55\right)$ (online Fig. DS6(a)) in the entire group with OCD $(n=46)$. In unmedicated participants with OCD $(n=23)$ only, significant positive correlations were again found between global OCD symptom severity and connectivity between the $\mathrm{dMPFC}$ and the ventral striatum $\left(\mathrm{MNI}_{x y z}=-10,14\right.$, $-10, z=4.02, r=0.74)$, whereas positive relationships between Y-BOCS scores and dMPFC connectivity with the anterior cingulate cortex were only evident at an uncorrected significance threshold of $P<0.001$ (online Fig. DS6(b)).

\section{Discussion}

\section{Main findings}

This study investigated the integrity of the default mode network subsystems in people with OCD using resting-state functional connectivity. Results revealed significantly reduced connectivity within the dMPFC self subsystem in the OCD group compared with a healthy control group, and further similar, but less pronounced effects in the midline core. In addition to abnormalities within the default mode network, the OCD group exhibited higher connectivity between components of the dMPFC self subsystem and the anterior insula and the superior parietal lobule, which represent central nodes of salience and dorsal attention networks, highlighting a general tendency towards higher connectivity with areas involved in active task engagement, which was evident in all three default mode network subsystems. Whereas indication of reduced midline core connectivity and higher interconnectivity among default mode and salience networks are in general agreement with the existing literature, these results provide first evidence of an aberrant default mode network subsystem in patients with OCD, and stronger interconnectivity of this subsystem with key regions of the salience and dorsal attention networks. Consequently, these findings point to multiple large-scale network involvement and suggest there should be careful examination of self-referential processing and activity of dMPFC self system components in patients with OCD in future studies.

\section{Findings from previous studies}

Previous studies provided evidence for either reduced connectivity within the default mode network ${ }^{11,12}$ or altered interconnectivity with other functional networks in OCD. ${ }^{13,14}$ Of note, a recent study found stronger connectivity between several default mode network regions and the anterior insula, ${ }^{14}$ which is highly consistent with the present confirmatory seed-to-voxel results conducted for a seed in this region (online Fig. DS5(b), online Table DS2). Similarly, trends for reduced posterior cingulate cortex connectivity with the midline core demonstrated by Stern et $a^{14}$ strongly converge with the present pattern of results observed for the midline core. In fact, seed-to-voxel differences for the midline core in the present study were only significant when considering the entire group of participants with OCD $(n=46)$ as compared with the control group, whereas only trends $(P<0.001$, uncorrected) were found for reduced midline core connectivity in unmedicated participants with OCD compared with the control group, suggesting that effects in the midline core might, to some extent, depend on sample size rather than medication status. In contrast, reduced connectivity in the dMPFC self system emerged in both seed-tovoxel and voxel-based region of interest (ROI)-to-ROI approaches (online supplement DS2), and remained significant after addressing potential effects of medication (online Table 4(a), online supplemental DS2). Similarly, exclusion of participants with a lifetime history of affective disorders revealed significantly reduced connectivity in seed-to-voxel analyses of the dMPFC self subsystem in the remaining participants with OCD (online Table DS5). This is worth noting because enhanced connectivity of the default mode network has previously been reported in patients with affective disorders. ${ }^{29}$ Taken together, the default mode network subsystem approach and the inclusion of a comparably large sample of people with OCD applied in the present study has allowed us to extend the existing literature by demonstrating both abnormal connectivity within the self subsystem of the default mode network and greater interconnectivity with other functional networks in the same sample of participants with OCD.

\section{Connectivity abnormalities in a neural system previously associated with self-referential processes in healthy individuals}

The finding of lower connectivity in the dMPFC self subsystem converges with evidence of reduced grey matter volume of the 
bilateral dMPFC in meta-analyses of structural MRI data of people with OCD. ${ }^{30}$ Studies in healthy individuals have elucidated a privileged role for the dMPFC subsystem in self-referential and affective processing. ${ }^{31-33}$ For example, the dMPFC is active when healthy participants judge whether emotional pictures make them feel pleasant or unpleasant, ${ }^{31}$ when judging whether personality traits accurately describe them ${ }^{32}$ and during simulation of personal events (in conjunction with midline core areas). ${ }^{18}$ In light of these findings closely associating activity of dMPFC self system components with self-referential processing, the present findings of reduced dMPFC self system connectivity could be interpreted to reflect altered self-referential processing in people with OCD. However, given the absence of behavioural measures reflective of self-referential thoughts or processes in the present study, this conclusion cannot be drawn from the present data and will require direct examination of people with OCD using experiments that have previously associated activity of dMPFC self system components and self-referential processes in healthy individuals. $^{18,33}$

From a clinical perspective, abnormal cognitive processing in relation to the self is frequently observed in OCD. ${ }^{1,34}$ More precisely, obsessions are usually ego-dystonic ${ }^{1}$ in the sense that their content is perceived as inconsistent with the patient's sense of self. ${ }^{34}$ Considering that the mental representation of the self and the degree of certainty with which a self-view is held are specifically mediated by the $\mathrm{dMPFC},{ }^{33}$ a compelling conclusion is that reduced connectivity in the $\mathrm{dMPFC}$ self system is related to impaired dissociation from obsessional content, because impaired recruitment of this system providing certainty of a self-view might facilitate the occurrence of obsessive, ego-dystonic thoughts. This interpretation receives support from the observation that relatively higher connectivity of the dMPFC with corticostriatal areas thought to mediate obsessions ${ }^{2}$ was associated with higher severity of obsessive-compulsive symptoms (online Fig. DS6). However, the assumptions made regarding this potential mechanism require study of the dynamic interactions between the dMPFC self subsystem regions and corticostriatal areas that are involved in the generation of obsessions, and determination of whether reduced dMPFC self subsystem connectivity at rest leads to aberrant activity in this subsystem in response to self-reflective stimuli in people with OCD. This appears plausible since spontaneous resting activity and task-related responses are strongly related. ${ }^{35}$

As briefly indicated above, connectivity of the dMPFC with striatal and cingulate areas positively correlated with global OCD symptom severity. Despite the fact that these regions play important roles in the functional neuroanatomy of OCD, ${ }^{2}$ and even though these results emerged in the entire OCD group as well as in medicated and unmedicated participants alone, based on voxel-wise analyses using corrections for multiple comparisons, it has to be emphasised that these post hoc analyses aimed to explore the clinical validity of the finding of reduced default mode network subsystem connectivity, and thus did not test a priori hypotheses. Further, the interpretation of these correlational findings in the context of the present study is complicated by the fact that the identified regions are outside the default mode network. The present findings indicate lower dMPFC self system connectivity and associate higher dMPFC connectivity with corticostriatal areas with more severe symptoms. The existing OCD neuroimaging literature points to corticostriatal hyperactivity and hyperconnectivity. Thus, one possibility could be that a stronger development of corticostriatal networks at the expense of deficient development of the default mode network occurs in people with OCD, and therefore, higher connectivity between the corticostriatal and default mode networks, or impaired segregation between the two, might relate to symptoms. However, this idea remains entirely speculative at this point, since testing it would require longitudinal rs-fcMRI assessments of, for example, individuals at risk of developing OCD.

\section{Abnormal default mode network interconnectivity with other large-scale brain networks}

In addition to the observation of reduced connectivity (control group $>$ OCD group) within the dMPFC self system and the midline core, default mode network whole-brain seed-to-voxel analyses also revealed predominantly stronger connectivity (OCD group $>$ control group) of the default mode network components with cortical areas known to actively participate in cognitive task execution, and which also represent components of previously identified rs-fcMRI networks. ${ }^{7-10}$ For example, the temporal pole was more connected to the superior parietal lobule of the dorsal attention network, and further the dMPFC showed greater connectivity with the anterior insula of the salience network, whereas the retrosplenial cortex was more connected to the inferior frontal gyrus of the executive control network, respectively. This notion is supported by confirmatory seed-to-voxel analyses using dorsal attention, executive control and salience network seed coordinates that have been previously described in the literature (see online supplement DS1, online Table DS2, online Fig. DS5). Thus, in addition to the identification of reduced connectivity in the $\mathrm{dMPFC}$ self system, our data support previous $\mathrm{rs}$-fcMRI findings of abnormal interconnectivity between brain networks in OCD, ${ }^{14,15}$ as well as the recently formulated hypothesis that psychiatric abnormalities might be associated not only with alterations in a single system, but rather involve aberrant interconnectivity among multiple networks. ${ }^{10}$ More precisely, the triple network model formulated by Menon suggests a central role for the anterior insula of the salience network, which is thought to assign salience to external stimuli or internal mental events, thus disengaging (for example self-referential processing or stimulus-independent mental activity of) the default mode network, while initiating stimulus-dependent processing of the executive control network. ${ }^{10}$ Given the present pattern of results, it is plausible to consider that greater connectivity of the anterior insula with the dMPFC subsystem and other default mode network areas might lead to an abnormally high or even constant disengagement and reduced connectivity of the dMPFC self system, resulting in insufficient recruitment of self-referential processes when dissociation from obsessional impulses is demanded.

\section{Divergent default mode network connectivity profiles in OCD $\boldsymbol{v}$. affective disorders}

As briefly indicated above, hyperconnectivity of the default mode network has been detected in affective disorders such as dysthymia $^{29}$ and major depression. ${ }^{36,37}$ These findings contrast with the present finding of reduced default mode network connectivity identified in the present study. The interpretation that people with OCD and people with affective disorders are characterised by divergent default mode network connectivity profiles receives further support from the observation that the finding of reduced connectivity in the dMPFC self system remained significant when considering only those participants with no history of affective disorders (online Table DS5), despite a substantial reduction in statistical power because of the exclusion. Considering that the default mode network is thought to engage in mental processes such as introspection or mindwandering, ${ }^{38}$ which involves moving away from externally focused thoughts while initiating internal, self-focused thoughts, these 
potentially diverging default mode network connectivity profiles of the two patient groups might reflect differences in introspective processes. Default mode network hyperconnectivity in affective disorders is thought to relate to excessive introspection and thus internal focus in the form of rumination, ${ }^{29}$ whereas externally focused thoughts related to the OCD symptom profile and greater attention to potentially threatening ${ }^{39}$ as well as novel stimuli ${ }^{40}$ characterise patients with OCD. To further investigate these aspects, default mode network connectivity profiles as well as behavioural measures of self-referential processing should be directly compared between participants with OCD and affective disorders in future studies.

\section{Limitations}

This study has several limitations. As briefly indicated above, the question of how the identified dMPFC self subsystem abnormalities during the resting-state contribute to OCD behaviour remains to be tested using approaches that consider both resting-state and experimental fMRI data. Notably, direct associations between dMPFC self system dysconnectivity and potential alterations in self-referential processing in people with OCD remain to be demonstrated, and will require study designs collecting both dMPFC self system connectivity parameters and measures of self-referential processing, and/or fMRI experiments involving manipulation of self-referential processes in people with OCD. Furthermore, connectivity abnormalities were identified using a purely correlative methodological procedure that provides no information about causal influences between brain regions.

In conclusion, the present study conducted a detailed investigation of default network integrity in participants with OCD by testing for abnormalities in subsystems of the default mode network in this patient group. Results identified reduced connectivity within the dMPFC self subsystem, which also showed greater interconnectivity with dorsal attention and salience networks. Furthermore, dMPFC connectivity with striatal and anterior cingulate cortex areas positively correlated with symptom severity. Therefore, the present results show that multiple brain networks contribute to the clinical phenotype of OCD. They further provide first evidence of a default mode network subsystem abnormality that suggests the need for careful examination of potentially altered self-referential processing in this disorder. These aspects require further study in order to advance neurobiological models of OCD.

\footnotetext{
Jan C. Beucke, MS, Department of Psychology, Humboldt-Universität zu Berlin, Berlin, Germany and Athinoula A. Martinos Center for Biomedical Imaging, Charlestown, Massachusetts, USA; Jorge Sepulcre, MD, PhD, Department of Radiology, Massachusetts General Hospital, Harvard Medical School, Boston and Athinoula A. Martinos Center for Biomedical Imaging, Charlestown, Massachusetts, USA; Mark C. Eldaief, MD, Department of Neurology, Brigham and Women's Hospital, Harvard Medical School, Boston, Massachusetts, USA; Miriam Sebold Hospital, Harvard Medical School, Boston, Massachusetts, USA; Miriam Sebold,
MS, Department of Psychology, Humboldt-Universität zu Berlin and Emotional Neuroscience Group, Charité Universitätsmedizin Berlin, Berlin, Germany; Norbert Kathmann, PhD, Department of Psychology, Humboldt-Universität zu Berlin, Berlin, Germany; Christian Kaufmann, MS, Department of Psychology, Humboldt-Universität zu Berlin, Berlin, Germany

Correspondence: Jan C. Beucke, MS, Department of Psychology, Humboldt-Universität zu Berlin, Rudower Chaussee 18, 12489 Berlin, Germany. Email: jan.beucke@hu-berlin.de

First received 21 Aug 2013, final revision 23 Jan 2014, accepted 24 Mar 2014
}

\section{Funding}

This work was supported by a grant from the Federal Ministry of Education and Research of Germany (grant number BMBF-01GW0724 to N.K.). J.C.B. is supported by a PhD scholarship from Evangelisches Studienwerk e.V. Villigst (Schwerte, Germany), and is a scholarship from Evangelisches Studienwerk e.V. Villigst (Schwerte, Germany), and is a
ERP scholar of the German National Academic Foundation. J.S. is supported by the Alzheimer's Association (grant number NIRG-11-205690).

\section{Acknowledgements}

The authors are grateful to the Cognitive Neuroscience Lab at Harvard University and the Athinoula A. Martinos Center for Biomedical Imaging for providing space and tools for rs-fcMRI analyses and Koene Van Dijk (PhD) for assistance with head motion analyses. Further, we thank Eva Kischkel (PhD) and Rüdiger Spielberg (PhD) for clinical assessments, Susan, Schub (MS) for patient (MS) for assistance involving data acquisition, Thomas Pinkpank (MS) for technical assistance
(M) and acknowledge scanning facility access provided by Charité Universitätsmedizin Berlin.

\section{References}

1 Salkovskis PM. Obsessional-compulsive problems: a cognitive-behavioural analysis. Behav Res Ther 1985; 23: 571-83.

2 Saxena S, Brody AL, Schwartz JM, Baxter LR. Neuroimaging and frontalsubcortical circuitry in obsessive-compulsive disorder. Br J Psychiatry 1998; 173 (suppl 35): 26-37.

3 Harrison BJ, Pujol J, Cardoner N, Deus J, Alonso P, Lopez-Sola M, et al. Brain corticostriatal systems and the major clinical symptom dimensions of obsessive-compulsive disorder. Biol Psychiatry 2013; 73: 321-8.

4 Beucke JC, Sepulcre J, Talukdar T, Linnman C, Zschenderlein K, Endrass T, et al. Abnormally high degree connectivity of the orbitofrontal cortex in obsessive-compulsive disorder. JAMA Psychiatry 2013; 70: 619-29.

5 Menzies L, Chamberlain SR, Laird AR, Thelen SM, Sahakian BJ, Bullmore ET. Integrating evidence from neuroimaging and neuropsychological studies of obsessive-compulsive disorder: the orbitofronto-striatal model revisited. Neurosci Biobehav Rev 2008; 32: 525-49.

6 Greicius MD, Krasnow B, Reiss AL, Menon V. Functional connectivity in the resting brain: a network analysis of the default mode hypothesis. Proc Natl Acad Sci U S A 2003; 100: 253-8.

7 Seeley WW, Menon V, Schatzberg AF, Keller J, Glover GH, Kenna H, et al. Dissociable intrinsic connectivity networks for salience processing and executive control. J Neurosci 2007; 27: 2349-56.

8 Fox MD, Corbetta M, Snyder AZ, Vincent JL, Raichle ME. Spontaneous neuronal activity distinguishes human dorsal and ventral attention systems. Proc Natl Acad Sci U S A 2006; 103: 10046-51.

9 Sylvester $\mathrm{CM}$, Corbetta M, Raichle ME, Rodebaugh TL, Schlaggar BL, Sheline $\mathrm{Yl}$, et al. Functional network dysfunction in anxiety and anxiety disorders. Trends Neurosci 2012; 35: 527-35.

10 Menon V. Large-scale brain networks and psychopathology: a unifying triple network model. Trends Cogn Sci 2011; 15: 483-506.

11 Fitzgerald KD, Stern ER, Angstadt M, Nicholson-Muth KC, Maynor MR, Welsh RC, et al. Altered function and connectivity of the medial frontal cortex in pediatric obsessive-compulsive disorder. Biol Psychiatry 2010; 68: 1039-47.

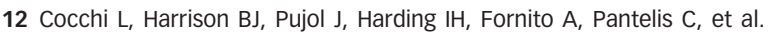
Functional alterations of large-scale brain networks related to cognitive control in obsessive-compulsive disorder. Hum Brain Mapp 2012; 33: 1089-106.

13 Jang JH, Kim JH, Jung WH, Choi JS, Jung MH, Lee JM, et al. Functional connectivity in fronto-subcortical circuitry during the resting state in obsessive-compulsive disorder. Neurosci Lett 2010; 474: 158-62.

14 Stern ER, Fitzgerald KD, Welsh RC, Abelson JL, Taylor SF. Resting-state functional connectivity between fronto-parietal and default mode networks in obsessive-compulsive disorder. PLOS One 2012; 7: e36356.

15 Li P, Li S, Dong Z, Luo J, Han $\mathrm{H}$, Xiong $\mathrm{H}$, et al. Altered resting state functional connectivity patterns of the anterior prefrontal cortex in obsessive-compulsive disorder. Neuroreport 2012; 23: 681-6.

16 Fitzgerald KD, Welsh RC, Gehring WJ, Abelson JL, Himle JA, Liberzon I, et al. Error-related hyperactivity of the anterior cingulate cortex in obsessive-compulsive disorder. Biol Psychiatry 2005; 57: 287-94.

17 Marsh R, Horga G, Parashar N, Wang Z, Peterson BS, Simpson HB. Altered activation in fronto-striatal circuits during sequential processing of conflict in unmedicated adults with obsessive-compulsive disorder. Biol Psychiatry 2014; 75: 615-22.

18 Andrews-Hanna JR, Reidler JS, Sepulcre J, Poulin R, Buckner RL. Functional-anatomic fractionation of the brain's default network. Neuron 2010; 65: 550-62.

19 American Psychiatric Association. Diagnostic and Statistical Manual of Mental Disorder (4th edn) (DSM-IV). APA, 1994.

20 First MB, Spitzer RL, Gibbon M, Williams JBW. Structured Clinical Interview for DSM-IV Axis 1 Disorders. American Psychiatric Press, 1996.

21 Goodman WK, Price LH, Rasmussen SA, Mazure C, Fleischmann RL, Hill CL, et al. The Yale-Brown Obsessive Compulsive Scale. I. Development, use, and reliability. Arch Gen Psychiatry 1989; 46: 1006-11. 
22 Foa EB, Huppert JD, Leiberg S, Langner R, Kichic R, Hajcak G, et al. The Obsessive-Compulsive Inventory: development and validation of a short version. Psychol Assess 2002; 14: 485-96.

23 Spielberger CD, Gorsuch RL, Lushene RE. Manual for the State-Trait Anxiety Inventory. Consulting Psychologists Press, 1970.

24 Beck AT, Ward $\mathrm{CH}$, Mendelson M, Mock J, Erbaugh J. An inventory for measuring depression. Arch Gen Psychiatry 1961; 4: 561-71.

25 Montgomery SA, Asberg M. A new depression scale designed to be sensitive to change. Br J Psychiatry 1979; 134: 382-9.

26 Oldfield RC. The assessment and analysis of handedness: the Edinburgh Inventory. Neuropsychologia 1971; 9: 97-113.

27 Schmidt KH, Metzler P. Wortschatztest (WST) [Vocabulary Test]. Beltz, 1992.

28 Van Dijk KR, Sabuncu MR, Buckner RL. The influence of head motion on intrinsic functional connectivity MRI. Neurolmage 2012; 59: 431-8.

29 Posner J, Hellerstein DJ, Gat I, Mechling A, Klahr K, Wang Z, et al. Antidepressants normalize the default mode network in patients with dysthymia. JAMA Psychiatry 2013; 70: 373-82.

30 Radua J, Mataix-Cols D. Voxel-wise meta-analysis of grey matter changes in obsessive-compulsive disorder. Br J Psychiatry 2009; 195: 393-402.

31 Gusnard DA, Akbudak E, Shulman GL, Raichle ME. Medial prefrontal cortex and self-referential mental activity: relation to a default mode of brain function. Proc Natl Acad Sci U S A 2001; 98: 4259-64.

32 Fossati P, Hevenor SJ, Graham SJ, Grady C, Keightley ML, Craik F, et al. In search of the emotional self: an fMRI study using positive and negative emotional words. Am J Psychiatry 2003; 160: 1938-45.
33 D'Argembeau A, Jedidi H, Balteau E, Bahri M, Phillips C, Salmon E. Valuing one's self: medial prefrontal involvement in epistemic and emotive investments in self-views. Cereb Cortex 2012; 22: 659-67.

34 Purdon C, Clark DA. Metacognition and obsessions. Clinical Psychol Psychother 1999; 6: 102-10.

35 Fox MD, Snyder AZ, Zacks JM, Raichle ME. Coherent spontaneous activity accounts for trial-to-trial variability in human evoked brain responses. Nat Neurosci 2006; 9: 23-5.

36 Greicius MD, Flores BH, Menon V, Glover GH, Solvason HB, Kenna $\mathrm{H}$, et al. Resting-state functional connectivity in major depression: abnormally increased contributions from subgenual cingulate cortex and thalamus. Biol Psychiatry 2007; 62: 429-37.

37 Sheline YI, Barch DM, Price JL, Rundle MM, Vaishnavi SN, Snyder AZ, et al. The default mode network and self-referential processes in depression. Proc Natl Acad Sci U S A 2009; 106: 1942-7.

38 Buckner RL, Andrews-Hanna JR, Schacter DL. The brain's default network: anatomy, function, and relevance to disease. Ann N Y Acad Sci 2008; 1124 $1-38$.

39 Muller J, Roberts JE. Memory and attention in obsessive-compulsive disorder: a review. J Anxiety Disord 2005; 19: 1-28.

40 Ischebeck M, Endrass T, Simon D, Kathmann N. Auditory novelty processing is enhanced in obsessive-compulsive disorder. Depress Anxiety 2011; 28: 915-23.

\section{poems by doctors}

\section{Asylum - no more}

\section{Reg Parton}

Empty crumbling wards echo the sighs Of the countless souls possessed;

Their solemn footfalls no longer measure The length of their tormented lives In endless, aimless peregrination Along the overgrown pavements.

No more the thousand-fold, The regimented herding No more the human industry,

The impersonal caring care;

No more the sanctuary austere,

No more ... no more. .

And so the silent screams

Bear out the tragedy

Of hopes now dashed, abandoned;

The future offering but little

To ease the burden of pain and fear,

The alienation and the sorrow.

[1993] 